\title{
Hydrolysis of pretreated rice straw with surfactants at low cellulase dosage
}

\author{
Yunshan Liang ${ }^{1,2, a}$, Zhijun Huang ${ }^{1,2}$, Shuzhou Miao ${ }^{3}$, Xingzhong Yuan ${ }^{3, b}$, \\ Lihong Jiang ${ }^{1,2}$, Yufeng Zhu ${ }^{1,2}$, Zhi Wang ${ }^{1,2, c}$
}

${ }^{1}$ College of Bioscience and Biotechnology, Hunan Agricultural University, Changsha, 410128, China

${ }^{2}$ Hunan Provincial Key Laboratory of Rural Ecosystem Health in Dongting Lake Area, Changsha, 410128, China

${ }^{3}$ College of Environmental Science and Engineering, Hunan University, Changsha 410082, P.R. China

alyss3399@126.com, byxz@hnu.cn, cwangzspider@sina.com

Keywords: enzymatic hydrolysis; cellulase; surfactant; monorhamnolipid

Abstract: The effects of three surfactants (Tween 80, saponin and monorhamnolipid) on the hydrolysis of alkali pretreated rice straw by low dosage of cellulase were studied. The results indicated that with a relatively low cellulase dosage ( $4 \mathrm{FPU} \mathrm{g}^{-1}$ substrate), all surfactants were able to enhance the enzymatic hydrolysis, while the biosurfactant monorhamnolipid was demonstrated to be more active than Tween 80 and saponin. Monorhamnolipid at $0.006 \%$ increased reducing sugar yield by $23.15 \%$, and the maximal sugar yield was obtained within half of the time compare to the control required. The cellulase losed $67 \%$ of its activity in the control, while the activity lost ratios were $31 \% \sim 43 \%$ in the presence of the surfactants. The surfactants addition did not affect the $\mathrm{pH}$ of the hydrolysis system. This study provides an opportunity of decreasing enzyme dosage in hydrolysis of agricultural solid waste rich in cellulose.

\section{Introduction}

Cellulosic biomass from agricultural crop residues, grasses, wood and municipal solid waste represents an abundant renewable resource has attracted more and more attentions as a future source of biofuels. Annually, millions of tons of lignocellulosic wastes are produced worldwide ${ }^{[1-4]}$. Composting of agricultural solid waste is of a long history in China and other agricultural countries $^{[5-7]}$. However, it takes a long time for the waste to be decomposed completely as the high content of cellulose, which is of a complex structure and hard to degrade ${ }^{[8,9]}$. The low cellulase activity is another reason why the degradation of cellulose is slow ${ }^{[10]}$. Cellulase, which catalyses the hydrolysis of cellulose to D-glucose, is dependent on the types of cellulolytic microorganisms present in the compost matrix ${ }^{[11]}$. In the work of maturity assessment of compost from municipal solid waste $^{[12]}$, it was found that the cellulase activity decreased during the first week of composting, and then decreased again after the third week.

In order to improve the cellulose conversion, many researches have focused on optimizing the enzymatic hydrolysis process and enhancing cellulase activity ${ }^{[13]}$. Cellulase dosage of $10 \mathrm{FPU} \mathrm{g}^{-1}$ cellulose is often used in lab-scale studies as it provides a hydrolysis profile with high levels of glucose yield with reasonable time $(48-72 \mathrm{~h})$ and enzyme cost. Cellulase enzyme dosages in hydrolysis vary from 7 to $33 \mathrm{FPU} \mathrm{g}^{-1}$ substrate, depending on the types and concentrations of substrates $^{[14]}$.

The stimulatory effect of surfactant on the process for cellulose conversion provides a possibility to reduce the enzyme dosage ${ }^{[15-17]}$. However, most of researches were focused on the synthetic surfactants. Compared with the synthetic surfactants, the use of biosurfactants has been associated with less frequent inhibitory effects on bioconversion ${ }^{[18]}$. Biosurfactants present higher environmental compatibility and higher activity at extreme temperature, $\mathrm{pH}$ and salinity ${ }^{[19,20]}$. Another important result reported by Helle ${ }^{[21]}$ is that the surfactant effect is higher at low cellulase 
concentration. However, few researches have presented the effect of surfactants addition for hydrolysis of cellulose at relatively low enzyme dosage.

In the present study, two biosurfactants (saponin and monorhamnolipid) and one synthetic surfactant Tween 80 were adopted. The aim of this study is to investigate the possibility of surfactants application to accelerate the decomposition of organic solid waste rich in cellulose, or to improve the cellulase application efficiency in cellulose industries.

\section{Materials and methods}

\section{Substrate}

Rice straw grew and was harvested in the outskirts of Changsha city, central south of China. It was air-dried, and then ground to powder which is smaller than $0.6 \mathrm{~mm}$. The sample was stored at room temperature for the further use. The initial composition of rice straw was $39.39 \%$ cellulose, $27.38 \%$ hemicellulose, $5.89 \%$ lignin, and $27.34 \%$ others. Before enzymatic hydrolysis, rice straw was alkali pretreated to increase the exposure of polysaccharides to hydrolytic enzymes. Straw sample was treated with $2 \% \mathrm{NaOH}$ at $85^{\circ} \mathrm{C}$ for $1 \mathrm{~h}$. The solid cellulosic residues were collected and washed thoroughly with tap water to neutral $\mathrm{pH}$, then dried at $50^{\circ} \mathrm{C}$. The pretreated straw was analyzed for chemical components, and showed that it contains $69.28 \%$ cellulose, $13.37 \%$ hemicellulose, $4.91 \%$ lignin and others $12.44 \%$.

\section{Surfactants}

The nonionic surfactant Tween 80 of analytical grade was purchased from Chemicals, Tianjin, China. The natural surfactant Saponin with the total impurities of 8-25\% sapogenin was supplied by Sigma-Aldich, German. The monorhamnolipid used in this study was produced by $P$. aeruginosa AB93066 ${ }^{[22]}$. The Pseudomonas aeruginosa strain AB93066 was obtained from the China Center for Type Culture Collection (CCTCC), and maintained as slants on peptone agar medium at $4{ }^{\circ} \mathrm{C}$. It was transferred monthly. The CMC of the monorhamnolipid was determined from a semilog plot of surface tension versus monorhamnolipid concentration, and it was calculated to be $58 \mathrm{mg} \mathrm{L}^{-1}$.

\section{Enzymatic hydrolysis}

The hydrolysis was carried out in $100 \mathrm{ml}$ flasks, at $2 \%(\mathrm{w} / \mathrm{v})$ pretreated material concentration in $0.05 \mathrm{M}$ sodium citrate buffer ( $\mathrm{pH} 4.80$ ) using an enzyme dosage of 4 Filter Paper Units (FPU) $\mathrm{g}^{-1}$ substrate. The commercial cellulase was kindly provided by Beita Biotech co. lid, Shanghai, China with a filter paper activity of $583 \mathrm{FPU} \mathrm{g}^{-1}$, as measured by the filter paper assay ${ }^{[23]}$. The treatment without surfactant addition was used as control. The surfactants addition of Tween 80 and saponin each were used at $0.02 \%, 0.06 \%$, while the monorhamnolipid was tested at $0.006 \%$ and $0.018 \%$. Enzymatic hydrolysis was performed at $150 \mathrm{rpm}$ in a rotary shaker at $50^{\circ} \mathrm{C}$ for $72 \mathrm{~h}$. The reducing sugar yield calculated as amount of reducing sugar relative to the initial substrate concentration $(20 \mathrm{~g}$ $\mathrm{L}^{-1}$ ). The filter paper activity (FPA) of the supernatant was measured periodically during the hydrolysis. All experiments were performed in triplicate. Samples for sugar analysis were boiled for 5 min to terminate the reaction and stored at $-20^{\circ} \mathrm{C}$ until analysis.

\section{Analysis}

Reducing sugars were determined by the 3,5-dinitrosalicylic acid (DNS) method, and activity of cellulase was measured according to the method of Ghose ${ }^{[23]}$, and expressed in filter-paper units. One unit of enzyme was defined as the amount of enzyme capable of producing $1 \mu$ mol of reducing sugars in $1 \mathrm{~min}$. The $\mathrm{pH}$ value was determined used a pHSJ-3F pH meter (Shanghai, China). Chemicals used for the experiments and analyses were of analytical grade. 


\section{Results and discussion}

\section{Enzymatic hydrolysis}
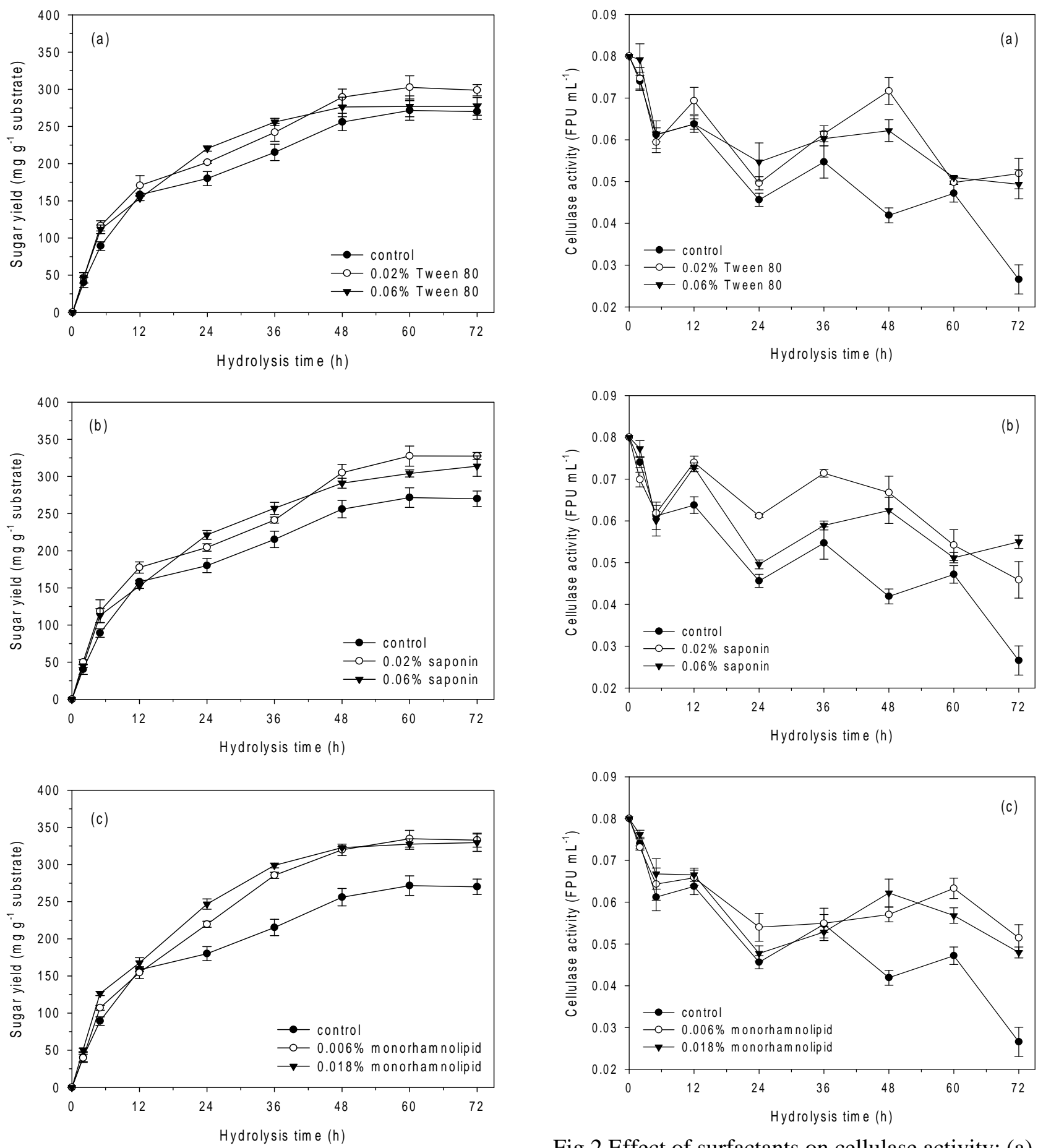

Fig.1 Effect of surfactants on the enzymatic hydrolysis: (a) Tween 80; (b) saponin; and (c) monorhamnolipid. The vertical bars designate the standard deviations for the mean of three replicative tests.

At the beginning of hydrolysis (0-12 h), the effect of surfactants was unobvious (Fig. 1). However, it was noticeable that the sugar yield markedly increased after $12 \mathrm{~h}$. In hydrolysis of straw without surfactant, $270.05 \mathrm{mg} \mathrm{g}^{-1}$ substrate of sugar yield was obtained after $72 \mathrm{~h} .0 .02 \%$ Tween 80 had a slight effect on the hydrolysis, resulting in $298.76 \mathrm{mg} \mathrm{g}^{-1}$ of reducing sugar yield obtained after $72 \mathrm{~h}$ hydrolysis (Fig. 1a). The effect of Tween 80 at the concentration of $0.06 \%$ was unremarkable. Both 
addition of saponin and monorhamnolipid improved the reducing sugar production remarkably (Fig.1 $\mathrm{b}$ and $\mathrm{c}$ ). After $72 \mathrm{~h}$ of hydrolysis, the reducing sugar yields were increased by $21.23 \%$ and $16.25 \%$ in the presence of $0.02 \%$ and $0.06 \%$ saponin, respectively. Monorhamnolipid at the $0.006 \%$ and $0.018 \%$ concentrations has similar effect, while $0.006 \%$ monorhamnolopid enhanced the sugar yield to $332.82 \mathrm{mg} \mathrm{g}^{-1}$ substrate with an increment of $23.24 \%$ compared with the control. In addition, it can be observed from the results that the maximal sugar yield occurred at about $60 \mathrm{~h}$ of hydrolysis without surfactant. However, the similar sugar yield (270 $\mathrm{mg} \mathrm{g}^{-1}$ substrate) was obtained at $42 \mathrm{~h}$ in the presence of Tween 80 or saponin, and at $30 \mathrm{~h}$ in the presence of monorhamnolipid, respectively.

Various explanations to the surfactant effect have been proposed including the increment of enzyme stability and the increasing accessibility of the substrate ${ }^{[15]}$. Other mechanisms include that surfactant is able to change the nature of the substrate, thereby increasing the availability of cellulose surface; in turn promoting more sites for cellulases to be adsorbed onto ${ }^{[21,24]}$. It is shown by the correlation between surfactant concentration and cellulose conversion that the higher concentration of surfactant does not always lead to the higher increment in cellulose conversion. It can be due to that all possible binding sites on substrate are occupied by surfactant which reaches a certain concentration, irrespective of the ability of substrate to unspecifically bind enzymes ${ }^{[16]}$. In other words, there may be several potential sites on the substrate that may either adsorb enzyme of surfactant. When these sites are all associated with surfactant, further addition will not increase hydrolysis.

\section{Effect of surfactants on enzyme stability}

The results on the stability of cellulase are presented in Fig.2, which shows marked effects of surfactants at a relatively low (4 FPU $\mathrm{g}^{-1}$ substrate) enzyme dosage. In the treatments without surfactant, the cellulase losed $67 \%$ of its activity, and showd lower than $0.03 \mathrm{FPU} \mathrm{mL}^{-1}$ after $72 \mathrm{~h}$ hydrolysis. While different concentrations of surfactants effectively preserved the enzyme activity, showed an average FPA of $0.05 \pm 0.005 \mathrm{FPU} \mathrm{mL}^{-1}$ after $72 \mathrm{~h}$. The activity lost ratios were between $31 \% \sim 43 \%$ distinguish by different category and concentration of added surfactant.

Enzyme stabilization is an important process in the field of micellar enzymology, since most of enzymes lose their activity after a few hours of incubation. Kim et al. ${ }^{[25]}$ showed cellulase deactivation by shear forces due to agitation of the hydrolysis mixture. The effect of adding non-ionic surfactants was proposed to be caused by reduced contact of enzyme with the air-liquid interface due to the surface activity of the surfactant. Eriksson [10] have reported that the addition of non-ionic and anionic surfactants to a hydrolysis mixture of cellulose resulted in a decrement of cellulase adsorption to the residual substrate, which prevents the enzyme from being deactivated by such irrevserble adsorption. On the other hand, this effect of surfactant is able to increase the possibility to recycle enzymes after complete cellulose hydrolysis. The enzyme recycling can effectively increase the rate and yield of the hydrolysis, and consequently, reduce the enzyme cost. The results from this research indicate that at a relatively low enzyme dosage of $4 \mathrm{FPU}^{-1}$ substrate, surfactants addition successfully inhibit the cellulase deactivation.

\section{Surfactants influence on pH}

Most of the studies on enzymatic hydrolysis of lignocellulosic biomass choose $\mathrm{pH}$ 4.0-5.5 as the initial $\mathrm{pH}$ value. It can be due to that the higher or lower $\mathrm{pH}$ value causes the enzyme denaturation. The results showed that the $\mathrm{pH}$ values of all samples were in the same range of $4.7 \pm 0.1$ after $72 \mathrm{~h}$ of enzymatic hydrolysis (data not shown). It means that the surfactants influence on the $\mathrm{pH}$ is negligible, which confirms the previous study by Shi ${ }^{[26]}$. 


\section{Conclusions}

The surfactants enhanced the reducing sugar yield during the $72 \mathrm{~h}$ hydrolysis at low enzyme dosage, and the biosurfactant monorhamnolipid was more effective than Tween 80 and saponin used in this research. All surfactants showed marked effects on the stability of cellulase during the hydrolysis process. The $\mathrm{pH}$ maintenance indicated that surfactants addition had no negative effect on the enzymatic hydrolysis of cellulosic waste.

\section{Acknowledgements}

This work was financially supported by the National Natural Science Foundation of China (No. 41401358) and China Postdoctoral Science Foundation (No. 2013M542112).

\section{References}

[1] B.L. Turner, S. Newman, and J.M. Newman: Environ. Sci. Technol. Vol. 40 (2006), p. 727-733

[2] M. Diego, M.B. Randy, H. Bernard, S. Markku, A. Mikko and E.B. Scott: Nat. Biotechnol. Vol. 26 (2008), p. 553-560

[3] N. Sarkar, S. K. Ghosh, S. Bannerjee and K. Aikat: Renew Energy Vol. 37 (2012), p. 19-27

[4] L.L. Zhang, H.Q. Zhang, Z.H. Wang, G.J. Chen and L.S. Wang: Bioresour. Technol. Vol. 203(2016),p.1-10

[5] M. Tuomela, M. Vikman, A. Hatakka and M. Itävaara: Bioresour. Technol. Vol. 72 (2000), p. $169-183$

[6] J.A. López-González, M.J. López, M.C. Vargas-García, F. Suárez-Estrella, M. Jurado and J. Moreno: Bioresour. Technol. Vol. 146(2013), p. 574-584

[7] J.A. López-González, M.D.C. Vargas-García, M.J. López, F. Suárez-Estrella, M.D.M. Jurado and J. Moreno: Bioresour. Technol. Vol. 187(2015), p. 305-313

[8] J. Pérez, J. Muňoz-Dorado, T. Rubia and J. Martínez: Int. Microbiol Vol. 5 (2002), p. 53-63

[9] X.X. Liua, Q. Xua, J.T. Liu, D.L. Yin, S.P. Su and H. Ding: Fuel Vol.164 (2016), p.46-50

[10]R.P. Tengerdy and G. Szakacs: Biochem. Eng. J. Vol. 13 (2003), p. 169-179

[11]S. Goyal, S.K. Dhull and K.K. Kapoor: Bioresour. Technol.Vol. 96 (2005), p. 1584-1591

[12]P. Castaldi, G. Garau and P. Melis: Waste Manage. Vol. 28 (2008), p. 534-540

[13]C. Zheng, Y. Lei, Q. Yu, X. Lui and K. Huan: Environ. Technol. Vol. 23 (2002), p. 1009-1016

[14]Y. Sun and J.Y. Cheng: Bioresour. Technol. Vol. 83 (2002), p. 1-11

[15]T. Eriksson, J. Börjesson and F. Tjerneld: Enzyme. Microb. Technol. Vol. 31 (2002), p. 353-364

[16]J.B. Kristensen, J. Börjesson, M.H. Bruun, F. Tjerneld and H. Jørgensen: Enzyme Microb. Technol. Vol. 40 (2007), p. 888-895

[17]K.N. Li, J.M. Wan, X. Wang, J.F. Wang and J.H. Zhang: Ind. Crops Prod. Vol. 83 (2016), p. 414-422

[18]Z.Y. Zhao and J.W.C. Wong: Environ. Technol.Vol. 30 (2009), p. 291-299

[19]C.N. Mulligan: Environ. Pollut. Vol. 133 (2005), p. 183-198

[20]K.Y. Cheng, Z.Y. Zhao and J.W.C. Wong: Environ. Technol.Vol. 25 (2004), p. 1159-1165

[21]S.S. Helle, S.J.B. Duff and D.G. Cooper: Biotechnol. Bioeng. Vol. 42 (1993), p. 611-617

[22] Y.S. Liang, X.Z. Yuan X Z, G.M. Zeng, C.L. Hu, H. Zhong, D.L. Huang, L. Tang and J.J. Zhao: Biodegradation. Vol. 21 (2010), p. 615-624

[23]T.K. Ghose: Pure. Appl. Chem. Vol. 59 (1987), p. 257-268

[24]W.E. Kaar and M. Holtzapple: Biotechnol. Bioeng. Vol. 59 (1998), p. 419-427

[25]M.H. Kim, S.B. Lee and D.D.Y. Ryu: Enzyme Microb. Technol. Vol. 4 (1982), p. 99-103

[26]J.G. Shi, G.M. Zeng, X.Z. Yuan, F. Dai, J. Liu and X.H. Wu: World J. Microbiol. Biotechnol. Vol. 22 (2006), p. 1121-1127 